\title{
The Effect of Multisensory Stimuli on Path Selection in Virtual Reality Environments
}

\author{
Guilherme Gonçalves ${ }^{1(\bowtie)}$, Miguel Melo², José Martins ${ }^{1,2,3}$, \\ José Vasconcelos-Raposo ${ }^{1,2}$, and Maximino Bessa ${ }^{1,2}$ \\ 1 University of Trás-os-Montes and Alto Douro, Vila Real, Portugal \\ guilhermeg@utad.pt \\ 2 INESC TEC, Porto, Portugal \\ 3 EsACT, Instituto Politécnico de Bragança, Bragança, Portugal
}

\begin{abstract}
Virtual Reality (VR) has as a key feature, the users' interaction with a virtual environment. Depending on the purpose of a given VR application, it can be essential to use multisensory stimulus without biasing users towards specific actions or decisions in the virtual environment (VE). The goal of the present work is to study if the choice of paths can be influenced by the addition of multisensory stimulus when navigating in a VE using an immersive setup. The awareness of having to take such decisions was also considered. For the purpose, we used a VR gamelike application contemplating three levels. Each level was symmetrical and had two possible paths to move to the next level (left or right). For each level, there was a multisensory stimulus on the right path (from a subject orientation): wind, vibration, scent respectively. The sample of the study consisted of 50 participants, and the results showed that none of the multisensory stimuli had a significant impact users' decision. The users' awareness of having to decide also did not affect their path. We conclude that multisensory stimuli can be used to raise the credibility of the virtual environments without compromising the users' decisions.
\end{abstract}

Keywords: Virtual Reality $\cdot$ Multisensory $\cdot$ Credibility $\cdot$

Decisions Awareness

\section{Introduction}

The concept of Virtual Reality (VR) has been around for many years. However, only recently immersive VR equipment became accessible to the general public. Since then, immersive VR evolved at a fast pace giving the opportunity for users to become the actors in such virtual environments (VE) [1]. By actors, we refer to the possibility of interaction with the VEs by using the same physical movements we would use in the real world. Such possibilities open new ways to use $\mathrm{VR}$ in application fields like education [2] or training [3,4]. In both areas, VR 
can be used to test or train concepts that require users to make the right decisions. It allows simulations of situations that could be difficult to mimic in real life regarding logistics, costs or safety. For example, in surgery, students could practice procedures repeatedly in a safe environment without compromising the health of real patients.

\section{$1.1 \quad$ Related Work}

The feeling of "being there" while in a VR application is known among the literature as Presence [5]. If a user feels high levels of presence, their behaviour in the VE should be more like the behaviour in a real analogous situation $[6,7]$. This fact suggests that higher levels of presence should be required to efficiently use VR applications to train or educate users to real-world situations. To achieve this feeling of presence, the VE should be credible [8]. Studies conducted proving multisensory increases presence [9], being that multisensory stimuli can play an important role.

Works suggest multisensory stimuli are essential for users to make choices or take decisions in certain situations [10]. Barbosa et al. [3] conducted an experiment where users use the haptic feedback of temperature to perform a firefighter procedure correctly. Regarding scents, those associated with dangerous situations can, for example, raise the level of awareness [11] and possibly influence how a user makes his decision. The correct use of multisensory cues can also manipulate the users' decisions without them knowing - e.g. using proper stimuli can increase how much users spend time and money in markets just by manipulating sounds, smells, colours and touch [12]. This fact suggests that these stimuli, even if we don't realise, can influence our decisions in real life.

From state of the art, we know that users' decisions can depend on the multisensory stimuli they receive. But in these cases, the multisensory stimuli were critical for the task at hand. For instance, users would have to be aware and constantly monitor the stimuli to decide based on it. To the best of our knowledge, no works could be found in the literature that studies how multisensory stimuli influences the path of users in immersive VR applications.

Our work investigates wherever the use of multisensory stimuli delivered in a subtle manner (not critical for the task), can influence users' decisions in VR. We will test each stimulus individually (wind, vibration, and smell) and analyse which path users take (left or right). Each stimulus will have its source on only one of the two paths. Another factor that we included in this study was if the knowledge of the fact that there is a decision to be made affects the decision itself. We theorise that if the player was not advised about the fact that he must decide between paths, he could opt randomly for the first path that he/she sees. Such could imply that users could not feel the stimuli coming from one of the sides if they went directly to the path without a multisensory stimulus associated. If the users know beforehand that there are two paths and they must choose one of them, we expect them first to analyse both paths and pay extra attention to possible differences between them. We then propose two hypotheses: 
- H1: The multisensory stimuli will influence the user's path.

- H2: The awareness of having to decide between paths will influence how they do it.

To test these hypotheses, we investigate in this work whether subtle multisensory cues and awareness about decision-making in VEs can influence the user's path when compared to the typical audiovisual VE setup.

\section{Methods and Materials}

An experimental cross-sectional study, of comparative nature following a between-group design was conducted to investigate the knowledge about the decision-making process and the impact of multisensory feedback in the user's path in VR applications as described below.

\subsection{Sample}

We used a non-probabilistic sampling technique, namely convenience sampling (sample taken from a group of people easy to reach). In this case, we've taken our sample from university students. The sample consisted of 50 participants (37 males and 13 females) aged between 17 and 44 years old $(M=24.88)$ and most were students. The participants were randomly divided into two main groups. In group $\mathrm{A}(N=25)$, participants did not know that they could make decisions in the VE. A researcher told participants in group $\mathrm{B}(\mathrm{N}=25)$ that they could make decisions in the VE. Each group was subdivided into 2 groups, resulting in a total of 4 groups. These subgroups consisted of 2 different conditions: one where participants experienced multisensory stimuli $(N=12)$ and one where experienced only audiovisual stimuli $(N=13)$.

\subsection{Materials}

For this experiment, the research team has developed an immersive VR application using Unity@ 2017, named "Illusions" that allowed to have full control over the study variables. The VR application is game-like and depicts a dark environment with a suspense component and composed by three levels. The levels are designed (Fig. 1) in such a way that the player must choose one of two possible paths to move through the next level.

In every level, the player must find a key to open a door in each and proceed to the next one (Left image on Fig. 2). The door to be unlocked is always situated in the middle of the paths. So, when opening the door, the player is always in the middle of the paths. In each level, both paths are symmetrical and look the same to avoid a possible bias towards a side [13] (Right image on Fig. 2). However, there is always a multisensory stimulus coming from the right path relative to the participant's orientation that can be felt in the middle. In the first level, there was a slight breeze coming from the right side. In the second level, there 
LEVEL 1

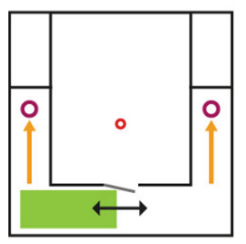

- STARTING POINT
LEVEL 2

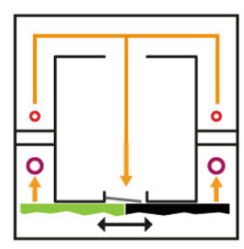

O NeXt LeVel
LEVEL 3

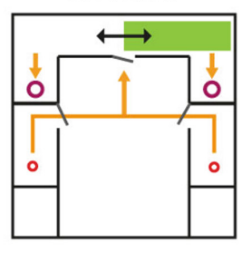

DECISION MAKING POINT

Fig. 1. Illusions level design

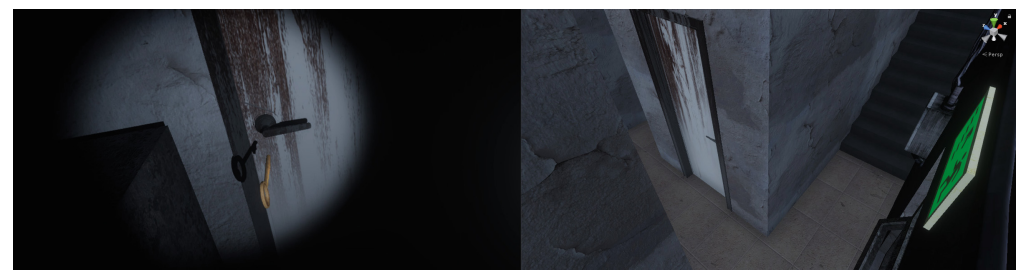

Fig. 2. Left: Player opening door after finding the hidden key. Right: Level 1 showing the left path (symmetrical to the right path)

was haptic feedback (vibration) from the ground beneath him that intensifies if he goes through the right side and that stops if he goes left. In the third and last level, the player could sense a burning scent coming from the right side of the level. However, there were problems with the users recognising the stimulus direction or even feeling the scent in the middle of the paths in the development stage of the game. To solve this, we implemented a game objective to fetch an object in the far end of both paths and go back to the middle so the subject could have a higher possibility to have sensed the scent in one of the sides while fetching the object. Based (or not) in this information the player could then choose the path to finish the game.

The levels were designed in a way that allows the player to walk physically through all the game in an area of 4 by $4 \mathrm{~m}$.

The experiments were run using a desktop computer with the following specifications: CPU Intel@ Core $^{\mathrm{TM}}$ i7-5820K @ $3.30 \mathrm{GHz}, 32 \mathrm{~GB}$ RAM, Geforce GTX 1080Ti. The visual stimulus, as well as the interaction, was ensured via the HTC VIVE system. Headphones with active noise cancelling were used to deliver audio and to isolate the participant from outside noises. A ground board delivered the force feedback stimuli in the ground with a mounted transducer. The wind stimulus was delivered using compressed air through a hose that was synced with the VE. For delivering olfactory stimuli, the SensoryCo SmX-4D smell machine was used. All the stimuli were synced with the VE. 


\subsection{Variables}

In this study, it was considered two independent variables: Multisensory Stimulus and Decision Knowledge. Regarding the Multisensory Stimulus, there were two levels: without multisensory stimulus (only visual and audio) and with the multisensory stimulus (visual and audio plus wind, force feedback, or smell). Regarding the Decision Knowledge, there were also two levels: without knowledge (participants were not informed that they would have to choose one from two possible routes) and with knowledge (participants were informed that they would have to pick one from two possible paths).

The dependent variable considered is the side (left or right) which the player decides to proceed to the next level.

\subsection{Procedure}

All the experiments were taken in an experimental room where the research team had full control over the ambient variables. When receiving the participants, they were briefed about the study - the purpose of the study was not disclosed to avoid bias. To formalise their agreement in participating in the study, they were asked to fill a consent. A generic sociodemographic questionnaire was also filled to be possible to characterise the sample (age and gender) - no sensitive personal data was collected as well as all the data was anonymous. As the experimental setup included a ground board that required participants to step on it (Fig. 3), participants were told to be careful with the ground obstacles as they could physically exist. This ground board was synced between the VE and reality, so the player could see this ground board in the game and step on it physically like it was real. To be sure players could sense the scent, we asked them if they had any limitations in breathing through the nose or detecting scents. If the response was positive, and to not compromise the experiment, players would perform conditions that did not consider multisensory stimuli.

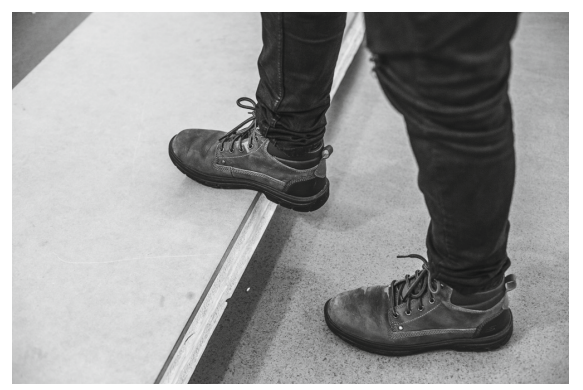

Fig. 3. Participant interacting with the ground board

Every participant began the experiment in the centre of the room, which corresponded to the centre of the game level, and in the same orientation. 
Throughout the whole experiment, the participants were always accompanied by a researcher to provide support if needed and to help the participants avoid the HMD cables. In each level of the game, players had to choose one of two possible routes to move through the next level. The VR application automatically logged the side the player selected.

The experiment ended when the player managed to reach the end of the game. The mean time that took for the participant to finish the game was around 14 min with the longest taking $22 \mathrm{~min}$ and the fastest $10 \mathrm{~min}$ to complete. After finishing the virtual experiment, a debriefing was conducted to collect data about the experiment (assess if participants had any factor in mind when deciding to go through one side or the other).

\subsection{Statistical Procedures}

Due to our dependent variable being dichotomic (left or right), we conducted a Pearson Chi-Square test to analyse if multisensory stimuli (namely wind, vibration, and scent) individually could influence participants to take one side more than the other. First, we performed the Pearson Chi-Square test between the groups A (W/ Multisensory) and B (W/ Multisensory) as well as between the groups A (W/O Multisensory) and B (W/O Multisensory) to verify if the variable Decision Knowledge affects how participants choose their path. If results reveal that this independent variable does not have any impact in the dependent variable, we will group the samples that experimented with multisensory stimuli across groups and compare it against the grouped sample that performed without multisensory stimuli.

\section{Results}

For ease of presentation, results are divided into two subcategories, corresponding to the two independent variables of the study: Decision Awareness and Multisensory Stimuli.

\subsection{Decision Awareness}

To verify if Decisions Awareness could influence the participants' path (H2), a Pearson Chi-Square test was performed for every level of the game to compare the side taken by the subjects between the multisensory condition (group A and B) and non-multisensory condition (group A and B).

Analysing the multisensory conditions, groups A (No knowledge) $(N=12)$ and $\mathrm{B}$ (With knowledge) $(N=12)$, results reveal that:

- For the first level (wind) no differences were found in the dependent variable Side $\left(\chi^{2}(1)=0.000, p=1.000\right)$. Both group A and B (W/ Multisensory), $50 \%$ of the participants $(N=6)$ went right and $50 \%$ went left $(N=6)$. 
- The second level (vibration) also had no differences $\left(\chi^{2}(1)=0.000 p=\right.$ 1.000). Similar to the first level, in both groups A and B (W/ multisensory) $50 \%$ of the participants $(N=6)$ chose the right path and $50 \%(N=6)$ the left one.

- The third level (scent) also revealed no differences between the conditions $\left(\chi^{2}(1)=1.510, p=0.219\right)$. In this level, group A had $66.7 \%$ of participants $(N=8)$ opting to go left, and $33.3 \%(N=4)$ to go right. Regarding group B, $41.7 \%$ of the participants $(N=5)$ went left and $58.3 \%(N=7)$ went right.

Analysing the non-multisensory condition, groups $\mathrm{A}(N=13)$ and $\mathrm{B}(N=$ 13), results revealed that:

- For the first level (wind) no differences were found between groups $\left(\chi^{2}(1)=\right.$ $0.000, p=1.000$ ). In both group A and B (W/O Multisensory) $69.2 \%$ of the participant $(N=9)$ chose to go left and $30.8 \%(N=4)$ to go right.

- The second level (vibration) revealed the same results, no differences between groups $\left(\chi^{2}=0.000, p=1.000\right)$. In both group $\mathrm{A}$ and $\mathrm{B}(\mathrm{W} / \mathrm{O}$ Multisensory) $46.2 \%$ of the participants $(N=6)$ chose to go left and $53.8 \%$ $(N=7)$ to go right.

- The third level (scent) also revealed no differences $\left(\chi^{2}(1)=2.476, p=\right.$ 0.116). In group A (W/O Multisensory) $30.8 \%$ of the participants $(N=4)$ went through the left path and $69.2 \%$ through the right path $(N=9)$. In group B (W/O Multisensory), $61.5 \%$ chose the left path $(N=8)$ and $38.5 \%$ the right one $(N=5)$.

\subsection{Multisensory Stimuli}

As no differences were found regarding the previous knowledge about having to decide one path to proceed to the next level, the samples were grouped by the two levels of the independent variable multisensory stimuli. A Pearson ChiSquare test between the conditions that involved multisensory $(N=24)$ and no multisensory $(N=26)$ was applied to verify if it can affect how subjects chose their path (H1) (Table1). The results revealed that:

- For the first level wind had no impact in the variable side $\left(\chi^{2}(1)=\right.$ 1.923, $p=0.166$ ). In the non-multisensory condition (Group A + B), $69.2 \%$ $(N=18)$ chose the left side and $30.8 \%(N=8)$ chose the right one. Regarding the multisensory condition (Group A + B), $50 \%(N=12)$ went right and $50 \%(N=12)$ went left.

- For the second level vibration also had no impact in the users decisions $\left(\chi^{2}=0.075, p=0.786\right)$. In the non-multisensory condition (Group A + B), $46.2 \%(N=12)$ chose the left side and $53.8 \%(N=14)$ chose the right one. Regarding the multisensory condition (Group A $+\mathrm{B}), 50 \%(N=12)$ went right and $50 \%(N=12)$ went left. 
Table 1. Percentage of participants that opted to go through the left or right paths based on Multisensory Stimuli

\begin{tabular}{l|l|l|l|l}
\hline \multirow{2}{*}{} & \multicolumn{2}{|l|}{ W/ Multisensory } & \multicolumn{2}{l}{ W/O Multisensory } \\
\cline { 2 - 5 } & Left & Right & Left & Right \\
\hline Level 1 & 50 & 50 & 69.2 & 30.8 \\
\hline Level 2 & 50 & 50 & 53.8 & 50 \\
\hline Level 3 & 54.2 & 45.8 & 54.2 & 58.3 \\
\hline Mean & 55.57 & 44.43 & 47.23 & 52.77 \\
\hline
\end{tabular}

- The third level revealed that scent also did not affect the dependent variable $\left(\chi^{2}(1)=0.321, p=0.571\right)$. In the non-multisensory condition (Group A + B), $46.2 \%(N=12)$ chose the left side and $53.8 \%(N=14)$ chose the right one. Regarding the multisensory condition (Group A + B), $54.2 \%(N=13)$ went right and $45.8 \%(N=11)$ went left.

\section{Discussion}

In this work, we studied how multisensory stimuli and previous knowledge about having to take a decision affects decision-making in VR. He hypothesised that multisensory stimuli, delivered in a subtle manner, (not critical for the task or objective) could influence participants path (H1). We also hypothesised that the awareness of the existence of two paths and having to decide between them could also influence the user's path (H2). Results demonstrated that none of the stimuli managed to influence the participant's path. We attribute these results to the fact that the stimulus, individually, was not important for the task or objective. For example, Spencer [10] says that the sense of smell has a vital role in both diagnosis and surgery. Thus, the sense of smell in this situation is important for the task, so users will pay more attention and make decisions according to the feedback of this stimuli. Another example is Barbosa et al. [3] work. It consisted in using temperature stimuli to perform a firefighter procedure. Here the user would also be aware of the presence of that stimuli and constantly seek temperate changes to perform the procedure correctly.

In this works the stimuli were important, and users would have to pay attention to it to perform the task and take the correct decisions. None of the stimuli we used (wind, vibration, smell) represented any strong importance in completing the game or any of the tasks of it. In other words, the game levels could be completed without the multisensory stimuli as easily as with them. From a gameplay perspective, these extra stimuli only helped to create an immersive environment. There was no challenge or objective associated with the multisensory stimuli. An example of a task which would require the user to pay attention to the stimuli and thus change how he/she acts could be: "The key that allows the player to unlock the doors and move through the next levels are always in the 
source of the multisensory stimuli". We also justify these results to the fact that some players did not explore both ways before going to the next level possibly not noticing that there was a difference which could influence the path.

Regarding the awareness of having to decide which path to take, results demonstrated no impact across conditions. From the debriefing session, we learned that none of the users told they went through one way because any multisensory stimuli attracted them, being the decisions random.

The results suggest that the use of multisensory stimuli in a subtle manner does not influence the user's path. This could prove useful, for example, if developers want to increase the credibility of a VE but they don't want it to influence the users' path. They could then add multisensory stimuli to archive that purpose - for example, a firefighter simulation where the researchers want the subjects to feel immersed and with high levels of presence. They could add multisensory stimuli to raise the credibility of the VE, but they don't want it to distract, overwhelm or influence the subjects out of their training steps.

\section{Conclusion}

The main objective of this study was to investigate multisensory stimulation and its impact regarding the user's path. The results suggested that multisensory stimuli did not have any effect on the subjects' path - also, the awareness of having to make a decision regarding the path they wanted to proceed revealed no differences when compared with the participants that were not aware of that. The study had some limitations. Different people can act differently to some of the stimulus (ex. the same scent can attract or repeal different persons) which could to a certain point influence the results). The last level had an extra objective that consisted in fetching an object in the far end of each path because it was impossible to determinate the direction and sometimes even sense the smell in the middle of the paths. This extra objective forced the player to experience both paths. This did not happen in the previous levels, and it could have influenced to some degree the results in that level. Also, the stimuli were always present to the right side of the subject in the moment of deciding which path to take which could have provoked some laterality bias.

The present work opens doors for further studies regarding the investigation whether the importance of the stimuli for the task at hand will influence the decisions of the users. Other stimuli, such as temperature, could also be incorporated into new studies. Also, the combination of multiple stimuli at the same time (ex. wind and temperature, or scent and wind) can be studied to verify its impact on the subject's path and decisions. The intensity of the stimuli could also be analyzed to understand from which threshold they can start to influence the user's paths and counterbalancing the stimulus positions to avoid a possible laterality bias. Also, the importance of the stimuli for the objectives should also be studied to investigate the points where subjects start to use the stimuli conscientiously to decide. 
Acknowledgement. This work is financed by the ERDF - European Regional Development Fund through the Operational Programme for Competitiveness and Internationalisation - COMPETE 2020 Programme and by National Funds through the Portuguese funding agency, FCT - Fundação para a Ciência e a Tecnologia within project POCI-01-0145-FEDER-031309 entitled "PromoTourVR - Promoting Tourism Destinations with Multisensory Immersive Media.

\section{References}

1. Fuchs, P., Moreau, G., Guitton, P.: Virtual Reality: Concepts and Technologies, 1st edn. CRC Press, London (2011)

2. Freina, L., Ott, M.: A literature review on immersive virtual reality in education: state of the art and perspectives. In: The International Scientific Conference eLearning and Software for Education, vol. 1, p. 133 (2015)

3. Barbosa, L., Monteiro, P., Pinto, M., Coelho, H., Melo, M., Bessa, M.: Multisensory virtual environment for firefighter training simulation: study of the impact of haptic feedback on task execution. In: 2017 24th Encontro Português de Computação Gráfica e Interação

4. Aïm, F., Lonjon, G., Hannouche, D., Nizard, R.: Effectiveness of virtual reality training in orthopaedic surgery. Arthrosc. J. Arthroscopic Relat. Surg. 32(1), 224$232(2016)$

5. Slater, M.: A note on presence terminology. Presence Connect 3 (2003)

6. Slater, M., Brogni, A., Steed, A.: Physiological responses to breaks in presence: a pilot study. In: Presence 2003: The 6th Annual International Workshop on Presence, vol. 157 (2003)

7. Bessa, M., Melo, M., de Sousa, A.A., Vasconcelos-Raposo, J.: The effects of body position on Reflexive Motor Acts and the sense of presence in virtual environments. Comput. Graph. 71, 35-41 (2018)

8. Bouvier, P.: The five pillars of presence: guidelines to reach presence. In: Spagnolli, A., Gamberini, L. (eds.) Proceedings of Presence, pp. 246-249 (2008)

9. Fröhlich, J., Wachsmuth, I.: The visual, the auditory and the haptic - a user study on combining modalities in virtual worlds. In: Virtual Augmented and Mixed Reality. Designing and Developing Augmented and Virtual Environments, pp. 159168. Springer. Heidelberg (2013)

10. Spencer, B.S.: Incorporating the sense of smell into patient and haptic surgical simulators. IEEE Trans. Inf. Technol. Biomed. 10(1), 168-173 (2006)

11. Köster, E.P.: The specific characteristics of the sense of smell. In: Rouby, C., Schaal, B., Dubois, D., Gervais, R., Holley, A. (eds.) Olfaction, Taste, and Cognition, pp. 27-44. Cambridge University Press (2002)

12. Soars, B.: Driving sales through shoppers' sense of sound, sight, smell and touch. Int. J. Retail Distrib. Manag. 37(3), 286-298 (2009)

13. Vilar, E., Rebelo, F., Noriega, P., Duarte, E., Mayhorn, C.B.: Effects of competing environmental variables and signage on route-choices in simulated everyday and emergency wayfinding situations. Ergonomics 57(4), 511-524 (2014) 